\title{
Testing PDR models against ISO fine structure line data for extragalactic sources
}

\author{
M. Vasta ${ }^{1}$, M. J. Barlow ${ }^{1}$, S. Viti ${ }^{1}$, J. A. Yates ${ }^{1}$ and T. A. Bell ${ }^{2}$ \\ ${ }^{1}$ Department of Physics and Astronomy, University College London, Gower Street, London \\ WC1E 6BT, UK. email: mv@star.ucl.ac.uk \\ ${ }^{2}$ Caltech, Department of Physics, MC 320- 47, Pasadena, CA 91125, USA
}

\begin{abstract}
Studies of our own Galaxy and observations of external galaxies have suggested that stellar ultraviolet radiation can ionize vast volumes of a galaxy and that far-ultraviolet radiation impinging on neutral cloud surfaces is responsible for a large fraction of the observed far-infrared (FIR) spectral line emission that cools the gas (Crawford \& al. (1985)). Fine structure (FS) emission lines can be used as tracers of nebular conditions such as density, excitation and ionization. By virtue of their different excitation potentials and critical densities, FS emission lines provide an insight into the energetics and chemical composition of the regions from which they originate. The far infrared [C II] $158 \mu \mathrm{m},[\mathrm{O}$ I] $145 \mu \mathrm{m}$ and [O I] $63 \mu \mathrm{m}$ fine structure emission lines obtained with the Infrared Space Observatory (ISO) from 35 extragalactic sources are examined to investigate the chemical abundances and large scales physical properties of these sources. Line fluxes are compared with a grid of PDR models previously computed using the UCL_PDR code. We overplotted our model predictions against flux ratios from the [C II] $158 \mu \mathrm{m}$ and $[\mathrm{O}$ I] $63 \mu \mathrm{m}$ and $145 \mu \mathrm{m}$ ISO LWS fluxes. In this section we will only discuss the sensitivity of the ratios to changes in the input parameters. We find that the average radiation field $\mathrm{G}_{0}$ is $60-8 \times 10^{2}$ and the average density $\mathrm{n}_{H} 10^{4}-9 \times 10^{4} \mathrm{~cm}^{-3}$. While ionised carbon, because of its ionisation potential, can be found in both neutral gas and ionised gas clouds, species such as ionised nitrogen [N II], with ionisation potential of $14.53 \mathrm{eV}$, can arise only from $\mathrm{H}$ II regions. The 11 sources that have detections of both [C II] $158 \mu \mathrm{m}$ and [N II] $122 \mu \mathrm{m}$ have mean and median $[\mathrm{C} \mathrm{II}]_{158} /[\mathrm{N} \mathrm{II}]_{122}$ flux ratios of 10.2 and 5.9 respectively. A H II region $[\mathrm{C} \mathrm{II}]_{158} /[\mathrm{N} \mathrm{II}]_{122}$ ratio of 1.6 implies that $\mathrm{H}$ II region contribute only $16 \%$ (mean case) and $27 \%$ (median case) of the overall [C II] $158 \mu \mathrm{m}$ flux that is observed. We used the above predicted $\mathrm{H}$ II region [C $\mathrm{II}]_{158} /[\mathrm{N} \mathrm{II}]_{122}$ ratio of 1.6 along with the observed [N II] $122 \mu \mathrm{m}$ fluxes, to correct the observed [C II] $158 \mu \mathrm{m}$ flux of these 11 sources for Hil region contributions. We estimate that $10-60 \%$ of the $[\mathrm{C} \mathrm{II}]$ is excited in ionised regions. When accounting for the contribution to the [CII] 158 $\mu \mathrm{m}$ by $\mathrm{H}$ II regions we found that our models fitted better the observations. We modeled the oxygen emission line profile emitted from an ensemble of PDRs and found a clear [O I] $63 \mu \mathrm{m}$ self-absorbed profile. We estimate that approximately $20-70 \%$ of the [O I] $63 \mu \mathrm{m}$ intensity may be suppressed through oxygen self-absorption depending on the physical parameters of the PDR regions. This work has been submitted for publication to MNRAS, Vasta et al. (2009).
\end{abstract}

Keywords. FIR fine structure emission lines, PDRs, Extragalactic Sources, ISO LWS, Oxygen self-absorption.

\section{References}

Crawford, M. K. Genzel, R. Townes, C. H., \& Watson, D. M 1980, ApJ755, 771 